\title{
A HYBRID TIME-FREQUENCY METHODOLOGICAL PROPOSAL TO SIMULATE GROUND RESPONSE UNDER HARMONIC ACCELERATIONS IN A MATERIAL-POINT FRAMEWORK
}

\author{
Cristian D. Rodríguez ${ }^{1}$, Luis. F. Prada-Sarmiento ${ }^{2 a}$, Torsten \\ Wichtmann $^{2 b}$ \\ ${ }^{1}$ Bauhaus-Universität Weimar \\ Coudraystraße 11c, 99423 Weimar, Germany \\ e-mail: cristian.rodriguez@uni-weimar.de \\ ${ }^{2}$ Ruhr-Universität Bochum \\ Gebäude IC Etage 5, 44801 Bochum, Germany \\ a email: luis.pradasarmiento@rub.de \\ b email: torsten.wichtmann@rub.de
}

Key words: Material point method, Fourier analysis, Seismic ground response, MPM-FEM coupling

\begin{abstract}
The response of geomaterials to seismic excitations, which are usually described with time history data, can be estimated by solving governing equations in the frequency domain and transferring quantities back to the time domain. However, one limitation of frequency analysis is the simplification of soil response by assuming constant stiffness during the action of seismic input. Therefore, when the frequency approach is used in ground response problems, linear-equivalent models of soil behaviour that allow the description of non-linear stiffness are implemented. In order to simulate large fields of displacements induced by seismic actions, this paper introduces a methodological proposal based upon a hybrid Finite Element (FE) time-frequency approach, coupled with the Material Point Method (MPM). In the FE solution, the soil stiffness changes after certain number of cycles and the equation of motion is solved in the frequency domain while the soil stiffness remains constant. Mapping of kinematic quantities between nodes of the finite element mesh and material points is performed via a Newton-Raphson numerical scheme. Each change of the stiffness matrix is marked by a convective materialpoint phase and the recalculation of material point locations. By following this approach, large deformations of geomaterials under constant amplitude harmonic accelerations can be simulated using a linear equivalent approach for the non-linear response. A model test case subjected to harmonic shaking is explained.
\end{abstract}

